\title{
Dual-specificity phosphatase 5 (DUSP5) as a direct transcriptional target of tumor suppressor p53
}

\author{
Koji Ueda ${ }^{1}$, Hirofumi Arakawa ${ }^{1,2}$ and Yusuke Nakamura*,1 \\ ${ }^{1}$ Laboratory of Molecular Medicine, Human Genome Center, Institute of Medical Science, University of Tokyo, 4-6-1 Shirokanedai, \\ Minato-ku, Tokyo 108-8639, Japan
}

\begin{abstract}
Dual-specificity phosphatase 5 (DUSP5), a VH1-like enzyme that hydrolyses nuclear substrates phosphorylated on both tyrosine and serine/threonine residues, has a potential role in deactivation of mitogen- or stressactivated protein kinases. Using cDNA-microarray technology, we found that the expression of DUSP5 mRNA was dramatically increased by exogenous p53 in U373MG, a p53-mutant glioblastoma cell line. Transcription of DUSP5 was also remarkably activated by endogenous p53 in response to DNA damage in coloncancer cells $(\mathrm{p53}+/+)$ that contained wild-type p53, but not in p53- $/-$ cells. Chromatin-immunoprecipitation (ChIP) and reporter assays demonstrated that endogenous p53 protein would bind directly to the promoter region of the DUSP5 gene, implying p53-dependent transcriptional activity. Overexpression of DUSP5 suppressed the growth of several types of human cancer cells, in which Erk1/2 was significantly dephosphorylated. If, as the results suggest, DUSP5 is a direct target of p53, it represents a novel mechanism by which p53 might negatively regulate cell-cycle progression by downregulating mitogen- or stress-activated protein kinases.
\end{abstract}

Oncogene (2003) 22, 5586-5591. doi:10.1038/sj.onc.1206845

Keywords: DUSP5; p53; Erk1/2

\section{Introduction}

Mutations in the $p 53$ gene are found in about half of all human cancers examined (Hollstein et al., 1991). The p53 gene product, a transcription factor, binds to its target genes in a sequence-specific manner. By regulating those transcriptional targets, p53 protein can protect cells from malignant transformation arising from severe DNA damage or critical cellular stress, by inducing either cell-cycle arrest or apoptosis (el-Deiry et al., 1992; Oren, 1994; Ko and Prives, 1996; Levine, 1997). To date,

\footnotetext{
*Correspondence: Y Nakamura; E-mail: yusuke@ims.u-tokyo.ac.jp ${ }^{2}$ Current address: Cancer Medicine and Biophysics Division, National Cancer Center Research Institute, 5-1-1 Tsukiji, Chuo-ku, Tokyo 104-0045, Japan

Received 22 January 2003; revised 15 May 2003; accepted 5 June 2003
}

dozens of such targets have been reported, but many remain to be identified.

Earlier we used two different methods to isolate p53target genes. One was differential display, using a cell line in which the expression of an exogenous wild-type p53 gene can be regulated under the control of the lactose operon. In this manner, we isolated five genes, TP53TG1 (Takei et al., 1998), TP53TG3 (Ng et al., 1999), fractalkine (Shiraishi et al., 2000), p53R2 (Tanaka et al., 2000), and p53DINP1 (Okamura et al., 2001). The other method was a yeast enhancer-trap system that allowed direct cloning of functional p53binding sequences from human genomic DNA (Tokino et al., 1994). By isolating and sequencing cosmid clones containing every p53-binding site obtained in that way, we identified four novel p53-target genes from genomic regions surrounding the binding sequences: GML (Furuhata et al., 1996), P2XM (Urano et al., 1997), BAI1 (Nishimori et al., 1997), and p53AIP1 (Oda et al., 2000).

The VH1 phosphatase encoded by a gene of vaccinia virus is a dual-specificity protein-tyrosine phosphatase (PTPase) that hydrolyses substrates phosphorylated on both tyrosine and serine/threonine residues. VH1-like PTPases have since been identified in humans and other organisms. Screening a human genomic library with a partial DUSPI cDNA yielded several novel PTPase genes, including one designated HVH3 (human VH1like PTPase-3) (Martell et al., 1994). HVH3, also called as DUSP5, was upregulated by TALL-1, a member of the family of tumor necrosis factors (Kovanen et al., 2002); this observation implied that induction of DUSP5 might deactivate mitogen- or stress-activated protein kinases. A recombinant protein containing the catalytic domain of DUSP5 has in fact displayed phosphatase activity toward ERK1 (MAPK3) in vitro (Ishibashi et al., 1994).

As a third approach to the search for p53 targets, we have been applying cDNA-microarray technology; such experiments have allowed us to document p53-dependent transcription of genes encoding Semaphorin3B (Ochi et al., 2002), CABC1 (Iiizumi et al., 2002), IRF5 (Mori et al., 2002b), and CyclinK (Mori et al., 2002a). Here, we report that the DUSP5 gene is yet another transcriptional target of $\mathrm{p} 53$, and show that it might be involved in p53-dependent suppression of cell growth. 


\section{Results and discussion}

To identify additional p53-target genes, we applied a cDNA-microarray technique to screen human cDNAs for p53-inducible transcripts, as previously described (McGrory et al., 1988; Ono et al., 2000). Transcription of the gene that encodes dual-specificity phosphatase 5 (DUSP5) was induced to a remarkable degree by the introduction of exogenous p53 into p53-deficient cells. The induction was confirmed on Northern blots, which indicated that the DUSP5 transcript was induced by overexpression of p53 using Ad-p53 (Figure 1a) and it was $2.5 \mathrm{~kb}$ long. We also examined whether endogenous p53 could activate the transcription of DUSP5 in coloncancer cell lines HCT116 (p53+/+) and HCT116 (p53-/-) in response to DNA damage. As shown in Figure 1b, the expression of DUSP5 mRNA increased remarkably in HCT116 $(\mathrm{p} 53+/+)$ cells, but not in HCT116 (p53-/-) cells, even though both cell lines had suffered genotoxic stress through adriamycin treatment or UV radiation. These results clearly suggested that DUSP5 was likely to be a strong candidate as a direct transcriptional target of p53.

Therefore we evaluated binding of the $\mathrm{p} 53$ protein to the transcriptional regulatory region of this gene by performing ChIP assays. We identified several candidate sequences as p53-binding targets in the promoter region and in intron 1, and then tested each of them for interaction with endogenous p53 protein. One potential p53-binding sequence (p53BS1), located $1.2 \mathrm{~kb}$ upstream of the transcription starting point (Figure 2a), was reproducibly present in the chromatin DNAs that were immunoprecipitated in endogenous p53-protein complexes, whereas none of the other fragments including p53BS2 was amplified by PCR using the same chromatin DNAs. We interpreted this result to mean that endogenous p53 protein had interacted with only p53BS1 in response to adriamycin-induced DNA damage (Figure 2b).
To examine further whether p53BS1 in fact has p53dependent transcriptional activity, we carried out reporter assays. A 331-bp genomic DNA fragment including the promoter and p53BS1 was cloned into pGL3-basic vector, and this construct (pGL3-p53BS1) was cotransfected into SaOS-2 cells along with wt-p53 or mt-p53 expression vectors. As shown in Figure 2c, luciferase activity was strikingly enhanced, up to nearly 50-fold, by co-transfection with wt-p53 expression vector, but not with mt-p53. Moreover, point mutations in the p53BS1 sequence (mt1) completely blocked this enhancement, suggesting that p53-dependent activity was specific to the sequence of p53BS1. These results, taken together, suggested that DUSP5 is a bona fide target of p53.

To explore the role of DUSP5 among the physiological functions of $\mathrm{p} 53$, we constructed a plasmid designed to express DUSP5 protein containing HA-tag at the N-terminus (N-HA-DUSP). N-HA-DUSP proteins were ectopically and stably expressed in A549 and H1299 lung-cancer cells, and in HCT116 p53 + / or p53-/- colon-cancer cells, by transfection of the $\mathrm{N}$ HA-DUSP plasmid (Figure 3a). As shown in Figure 3b, immunostaining experiments indicated that DUSP5 was localized in the nuclei of HCT116 (p53+/+) cells; similar results were obtained with A549, H1299, and HCT116 (p53-/-) cells (data not shown).

Using the same plasmid, we investigated a possible role of DUSP5 in cell growth by means of a colonyformation assay. We transfected pcDNA3.1 $(+) /$ DUSP5, pcDNA3.1(-)/DUSP5, or pcDNA3.1(+)/ into each of three cancer-cell lines (H1299, HCT116 p53+/ + , HCT116 p53-/-). The cells were cultured in the presence of G418, and the number of colonies was counted 2 weeks later. As shown in Figure 4a, enforced expression of DUSP5 significantly reduced the number of colonies in all the three cancer cell lines compared with the same lines using pcDNA3.1(-)/DUSP5 or pcDNA3.1 $(+) /$ vector, suggesting a growth-suppressive

a
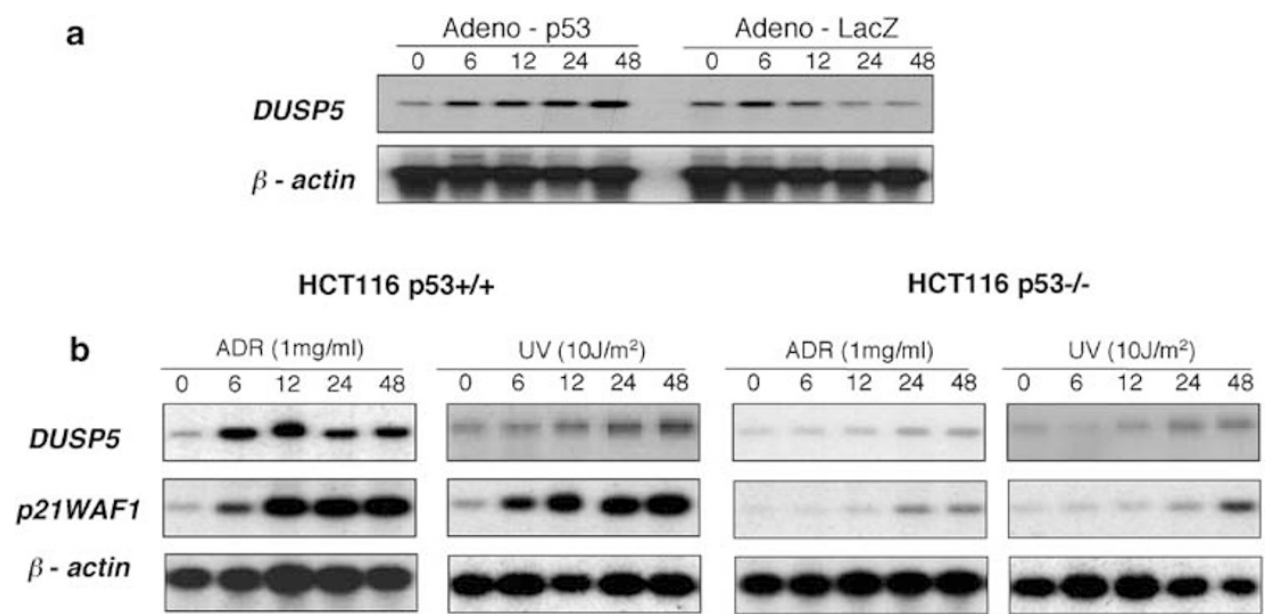

Figure 1 (a) Induction of DUSP5 and $\beta$-actin mRNAs in the human glioblastoma cell line U373MG by overexpressed p53, and (b) expression of DUSP5 mRNA by endogenous p53 after cytotoxic damage, as shown by Northern blotting. Expression of the $\beta$-actin gene served as a quantity control. DUSP5 mRNA was induced in cells infected by Ad-p53, but not in cells infected by Ad-LacZ (data not shown). DUSP5 and $p 21^{\text {wafl }}$ mRNAs increased with time following adriamycin or UV treatment in HCT116 p53 $+/+$ cells, but not in the HCT116 cell line lacking wild-type p53 
a
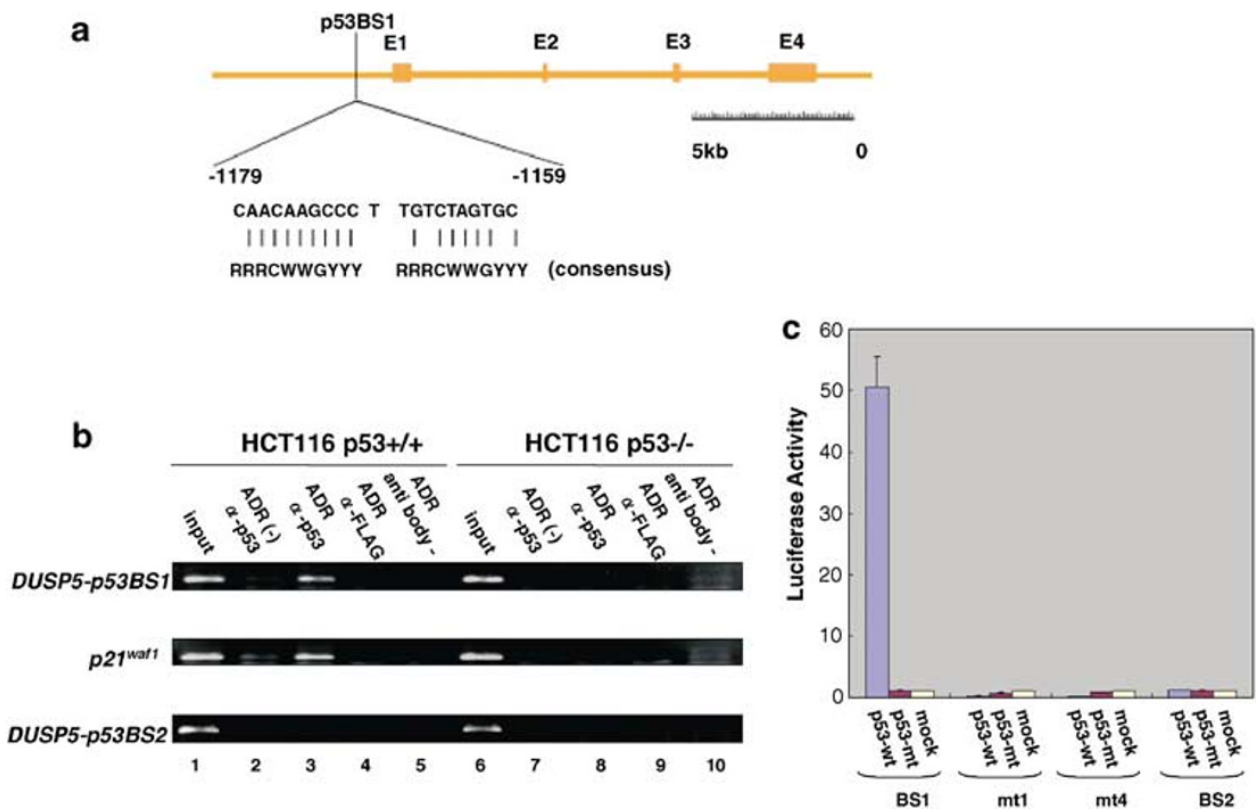

Figure 2 (a) Genomic structure of DUSP5. A potential p53-binding site is present in the promoter region, between nucleotide positions -1179 and -1159 , where +1 represents the translation-initiation site. (below) Sequence of the putative p53BS1-binding site compared to the consensus sequence. R, purine; Y, pyrimidine; W, A, or T. E1-E4 represent exons. (b) ChIP assay of a genomic fragment, nucleotide positions -1359 to -1028 containing the p53BS1 sequence, in damaged HCT116 p53 + / or HCT116 p53-/cells. After treatment with adriamycin, immunoprecipitation was performed using anti-p53 antibody (lanes 3 and 8 ). Input chromatin represents a portion of the sonicated chromatin prior to immunoprecipitation (lanes 1 and 6). Immunoprecipitates with an anti-FLAG antibody (lanes 4 and 9) or in the absence of antibody (lanes 5 and 10) served as controls. Immunoprecipitation with anti-p53 antibody was also performed using untreated cells (lanes 2 and 7). Amplification of the p21 promoter was performed for comparison, using primers flanking the p53-binding site of that gene. One of the other candidate sequences, nucleotide positions +871 to +1231 , was amplified only in the input control. (c) Responsiveness of the DUSP5 p53BS to p53. Reporters containing a 332-bp genomic sequence from the promoter region including p53BS (BS1) were constructed. One or four point mutations were inserted into p53BS (mt1 or $\mathrm{mt4}$ ). Activity is represented as a relative value of measured luminescence from firefly luciferase versus Renilla luciferase from cotransfected pRL-CMV (internal control). Mean values of the results of three experiments are displayed with error bars showing standard deviations

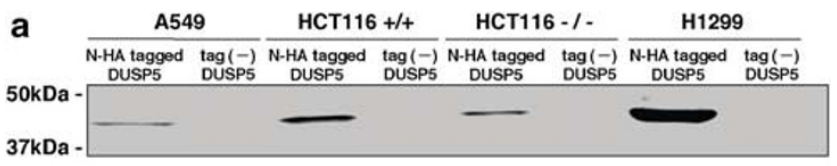

b
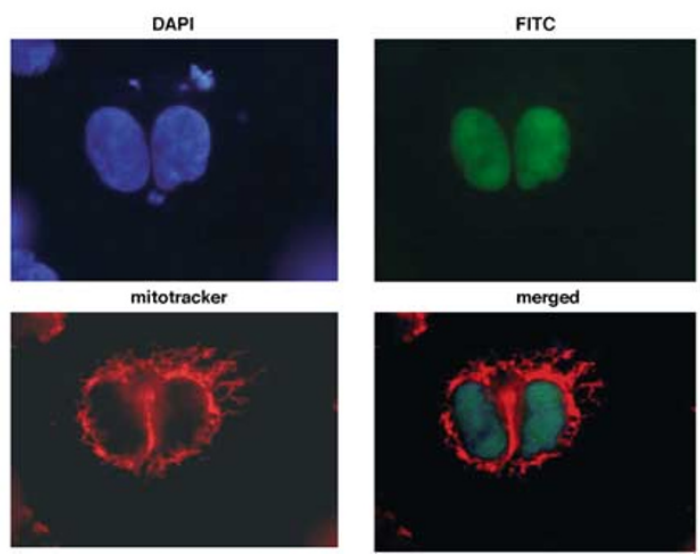

Figure 3 (a) Expression of DUSP5 in human cancer cell lines A549, HCT116 (p53+/+), HCT116 (p53-/-), or H1299 was confirmed by Western blotting using HA-tagged expression vectors containing the entire coding sequence of DUSP5. (b) Localization of DUSP5 in HCT116 (p53+/+) cells stained with anti-HA antibody (FITC), anti-human nucleus antibody (DAPI), and antihuman mitochondria antibody (mitotracker). Cells were strongly stained in the intranuclear region. The same results were obtained using HCT116 p53-/- and A549 cells (data not shown) role of DUSP5. In these experiments, the expression level of DUSP5 mRNA in each dish was confirmed by RT-PCR at 1 week after starting G418 selection. As shown in Figure 4b, DUSP5 mRNA was stably expressed in the cells transfected with pcDNA3.1(+)/DUSP5.

Cell-cycle regulation and apoptosis are the most important features of $\mathrm{p} 53$-dependent tumor suppression, each of those mechanisms being mediated by several p53-target genes. For example, p2 $1^{\mathrm{WAF} 1}$ is a pivotal mediator for arrest of the cell cycle at G1 phase, and BAX and p53AIP1 mediate p53-dependent apoptosis (Miyashita and Reed, 1995; Oda et al., 2000). In this study, we found DUSP5 to be a p53-target whose transcription was directly regulated by p53 in response to cytotoxic stress, and we demonstrated that overexpression of DUSP5 in several cancer cell lines triggered suppression of cell growth. However, the effect was unlikely to be due to either cell-cycle arrest or induction of apoptosis because we observed no remarkable changes with respect to those phenomena in our transient-transfection assay. The results suggested instead that another $\mathrm{p} 53$-dependent pathway to suppress growth of cancers was operating.

A recombinant protein containing the catalytic domain of DUSP5 has displayed strong phosphatase activity toward mitogen-activated protein kinase 3 (MAPK3) (Ishibashi et al., 1994). Moreover, related 

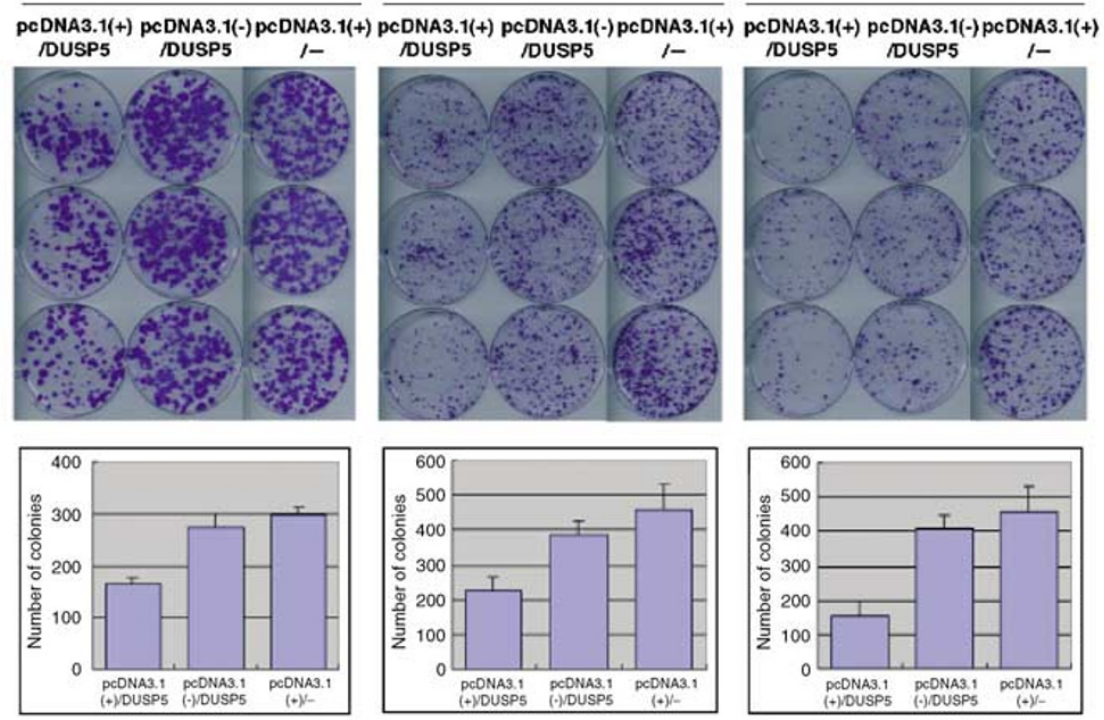

b

H1299

HCT116 p53+/+

HCT116 p53-\%

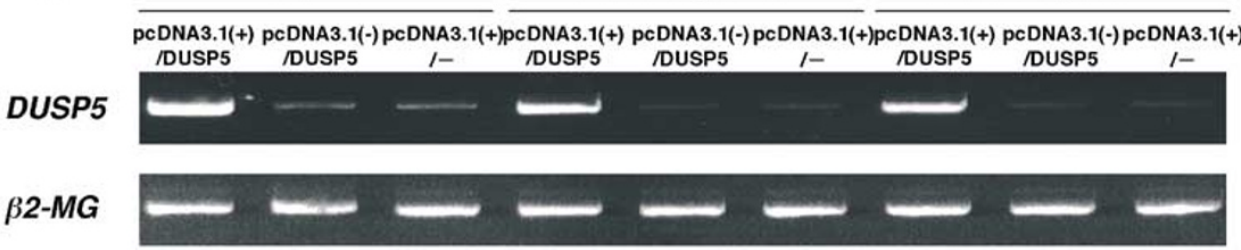

Figure 4 (a) Colony-formation assay. G418-resistant colonies grown from H1299, HCT116 p53 +/+, or HCT116 p53-/- cells transfected with either pcDNA3.1(+)/DUSP5, pcDNA3.1(-)/DUSP5, or pcDNA3.1 $(+) /$ were counted. The experiments were performed three times using triplicate samples, and average scores are indicated with error bars on the histogram below. (b) The expression of DUSP5 mRNA in the transfected cell lines. The result of RT-PCR indicated the expression levels of DUSP5 mRNA in H1299, HCT116 p53 +/+, and HCT116 p53-/- cell lines at 1 week after starting G418-selection. Expression of the $\beta 2-M G$ gene served as a quantity control

phosphatases such as DUSP1, DUSP4, and DUSP14 are also known as MAP kinase phosphatase 1 (MKP1), MKP2, and MKP6, respectively (Guan and Butch, 1995; Brondello et al., 1999; Marti et al., 2001). These facts prompted us to speculate that the growthsuppressive effect of DUSP5 might be mediated by its phosphatase activity toward mitogen-activated protein kinases including MAPK3. Suppression of cell growth in that case could reflect elongation of the cell cycle through dephosphorylation of MAPK3, which activates endogenous ribosomal transcription, while inactivation of MAPK3 would cause an immediate reversion to the basal transcription level (Stefanovsky et al., 2001).

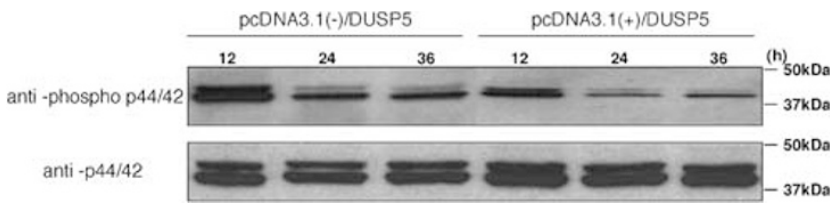

Figure 5 DUSP5 deactivates MAP kinases. Western blot analysis of p44/42 (Erk1/2) MAPK with anti-p44/42 and/or anti-phospho $\mathrm{p} 44 / 42$ in H1299 cells transfected with either pcDNA3.1(-)/DUSP5 or pcDNA3.1(+)/DUSP5 at the indicated times after transfection
Mitogen-activated protein kinases are often activated in human cancers, resulting in the transformation of cells and progression of the cell cycle (Kim et al., 2002). Point mutations in members of the ras family in particular, which are often found in colorectal and pancreatic cancers, are known to activate the pathway of mitogen-activated protein kinases and trigger malignant transformation of normal cells (Yoo et al., 2002).

We investigated whether DUSP5 actually had a phosphatase activity toward MAP kinases in the transient-transfection assay. The result of Western blotting was shown in Figure 5. Our result indicated that overexpression of DUSP5 indeed induced dephosphorylation of p44/42 (Erk1/2) in H1299 (Figure 5), suggesting that deactivation of MAP kinase might be involved in the mechanism of growth suppression of these cell lines.

Our discovery could provide a new model for p53dependent tumor suppression, in that through transcriptional activation of DUSP5 the $\mathrm{p} 53$ protein might play a pivotal role in deactivating the MAPK pathway, thereby slowing cell growth. A balance between the p53 and MAPK pathways appears to be critical for preventing or promoting initiation of tumors. 


\section{Materials and methods}

\section{Cell lines}

Human-cancer cell lines U373MG (glioblastoma), SaOS-2 (osteosarcoma), and A549 (lung cancer) were purchased from the American Type Culture Collection (ATCC, Manassas, VA, USA). Two colon-cancer cell lines, HCT116 p53+/+ and HCT116 p53-/-, were gifts from Bert Vogelstein. All cells were cultured under conditions recommended by their respective depositors.

\section{Detection of genes induced by $p 53$}

Adenoviruses containing p53 cDNA (Ad-p53) or $\beta$-galactocidase cDNA (Ad-LacZ) under control of the human cytomegalovirus promoter were generated as described previously (Ochi et al., 2002). U373MG cells, which lack wild-type p53, were infected with Ad-p53 or Ad-LacZ at 80 multiplicities of infection (MOI). Cells were collected 0, 6, 12, 24, and $48 \mathrm{~h}$ later, and the total RNA was extracted with TRIzol reagent (Life Technologies, Rockville, MD, USA). Poly(A) RNA was purified from each RNA sample using an mRNA purification kit (Amersham, Piscataway, NJ, USA). Each poly(A) ${ }^{+}$RNA was then amplified using Ampliscribe T7 Transcription Kits (Epicentre Technologies, Madison, WI, USA).

Genes induced by exogenous p53 were identified by hybridizing the RNAs to a cDNA microarray. Preparation of probes, hybridization procedures, and scanning were performed as described previously (Iiizumi et al., 2002). The fluorescent intensities of Cy5 (Ad-p53) and Cy3 (Ad-LacZ) for each target spot were adjusted so that the mean $\mathrm{Cy} 5 / \mathrm{Cy} 3$ ratio of each slide became equal. One induced gene, DUSP5, was selected from the microarray for further investigation.

\section{Northern blotting}

We prepared samples for Northern blotting analysis using U373MG cells that were infected by Ad-p 53 or Ad-LacZ for 0 , $6,12,24$, and $48 \mathrm{~h}$ and cells were used for Northern blots to confirm the results of cDNA-microarray analysis. Then, to check endogenous induction of DUSP5 in response to DNA damage, HCT116 p53+/ + and p53-/- cells were collected 0 , $6,12,24$, and $48 \mathrm{~h}$ after exposure to adriamycin $(1 \mu \mathrm{g} / \mathrm{ml})$ or ultraviolet irradiation $\left(10 \mathrm{~J} / \mathrm{m}^{2}\right)$. The total RNA was extracted with TRIzol reagent (Life Technologies, Rockville, MD, USA) and poly(A) ${ }^{+}$RNA was purified twice from total RNA with mRNA purification kits (Amersham, Piscataway, NJ, USA). A $2-\mu$ g aliquot of each poly(A) ${ }^{+}$RNA was separated on $1 \%$ agarose gel containing $1 \times 4$-morpholinepropane-sulfonic acid (MOPS) and $6 \%$ formaldehyde, and transferred onto a nylon membrane. Prehybridization and hybridization were carried out in solutions containing $50 \%$ formamide, $5 \times$ Denhardt's solution, $6 \times \mathrm{SSC}$, and $1 \%$ salmon-sperm DNA. The probes, carrying coding sequences of DUSP5, p21 WAF1, or $\beta$-actin genes, were labeled with gamma ${ }^{32} \mathrm{P}$-dCTP using a random primer labeling kit (Megaprime, Amersham Pharmacia Biotech). The membranes were hybridized with the radioactive probes at $42^{\circ} \mathrm{C}$ for $16 \mathrm{~h}$, washed, and exposed for autoradiography at $-80^{\circ} \mathrm{C}$.

\section{Chromatin-immunoprecipitation (chip) assay}

ChIP assays were performed using the Chromatin-immunoprecipitation Assay Kit (Upstate Biotechnology) as recommended by the manufacturer, except that we used antibodies against p53 (Ab-12; Oncogene) and FLAG-tag peptide (M2; Sigma Aldrich). HCT116 p53+/+ or p53-/- cells $\left(2 \times 10^{6}\right.$ each) were plated onto $10-\mathrm{cm}$ dishes and exposed to $1 \mu \mathrm{g} / \mathrm{ml}$ adriamycin for $2 \mathrm{~h}$. After $48 \mathrm{~h}$ of incubation in normal (adriamycin-free) medium, histones were cross-linked to DNA by addition of formaldehyde ( $1 \%$ final concentration) directly to the culture medium and the dishes were incubated at $37^{\circ} \mathrm{C}$ for $10 \mathrm{~min}$. Cells were lysed in $200 \mu \mathrm{l}$ of SDS lysis buffer with a protease-inhibitor mixture and sonicated to generate DNA fragments 200-1000 bp long. After centrifugation, the supernatant was diluted 10-fold with ChIP dilution buffer and incubated with the specific antibody preparations at $4{ }^{\circ} \mathrm{C}$ for $2 \mathrm{~h}$ with rotation. Immune complexes were precipitated, washed, and eluted as recommended. Histone-DNA crosslinks were reversed by heating at $65^{\circ} \mathrm{C}$ for $4 \mathrm{~h}$, and DNA was eluted from DNA-protein complexes by $20 \mu \mathrm{g}$ proteinase $\mathrm{K}$ solution at $45^{\circ} \mathrm{C}$, for $1 \mathrm{~h}$. Then DNA was extracted in phenol, precipitated with ethanol, and resuspended in $30 \mu \mathrm{l}$ of TE. A 1$\mu \mathrm{l}$ aliquot of each sample was used as a template for PCR. Amplifications were performed on immunoprecipitated chromatin using the specific primers 5'-AAGGCCCCCGCA TCACGTCTTC-3' and 5'-GTCAGCCGTGTGCCAGGGCTTT-3' for p53BS1, which corresponded to $332 \mathrm{bp}$ of the promoter region including a predictive $\mathrm{p} 53$-binding site; $5^{\prime}$ ACCTTTCACC ATTCCCCTAC-3' and 5'-GCCCAAGGACAAAATAGCCA-3' for p21 promoter; or 5'-GACTGCTCCTGCTTTGTGTTTG- $3^{\prime}$ and $5^{\prime}$-GGGGGA TAGGCTGTTAG AAGAA-3' for p53BS2.

\section{Construction of reporter plasmids}

A pGL3-p53BS1 reporter plasmid was constructed by cloning p53BS1 into PGL3-Basic vector (Promega). Then pGL3-mt1 or pGL3-mt4, containing, respectively, one or four nucleotideexchanged mutations in the consensus sequence for p53binding sites, were generated with QuickChange site-directed mutagenesis kits (Stratagene, La Jolla, CA, USA) using PCR primers $5^{\prime}$-GTTGTCAATAAGCCCTTGTCTAGTGCGGGC$3^{\prime}$ and $5^{\prime}$-GCCCGCACTAGACAAGGGCTTATTGACAAC- $3^{\prime}$ for pGL3-mt 1 or $5^{\prime}$ - CCCTTATGTCAGTTGTCAAAAATCC CTTGTATATTGCGGGCAGAC- $3^{\prime}$ and $5^{\prime}$-GTCTGCCCGC AATATACAAGGGATTTTTGACAACTGACATAAGGG$3^{\prime}$ for pGL3-mt4. All plasmid constructs were confirmed by DNA sequencing.

\section{Reporter-gene assays}

SaOS- 2 cells were plated in six-well culture plates $\left(2 \times 10^{5}\right.$ cells well) $24 \mathrm{~h}$ before co-transfection with wild-type p53 or $250 \mathrm{ng}$ of pGL3-BS, pGL3-mt1, or pGL3-basic vector, mutant-type p53 expression vectors, or pcDNA3.1 $(+)$ vector (wt-p53, mtp53, or mock) in combination with $70 \mathrm{ng}$ of pRL-CMV vector (Promega). At $48 \mathrm{~h}$ after transfection, the cells were lysed in $500 \mu \mathrm{l}$ of a passive lysis buffer. Cell lysates were directly forwarded to the luciferase assay. Quantification of luciferase activities and calculation of relative ratios were carried out manually with a luminometer.

\section{Expression of DUSP5 in human cancer cell lines}

We constructed a plasmid, pcDNA3.1(+)/N-HA-DUSP5, designed to express DUSP5 with an HA tag at the N-terminal end. First, Western blotting was performed to check the expression of DUSP5. Cells were plated in six-well culture plates $\left(1 \times 10^{5}\right.$ cells/well $) 24 \mathrm{~h}$ before transfection of pcDNA3.1 $(+) /$ N-HA-DUSP5 into A549 or H1299 cells (human lung-cancer lines), HCT116 p53 + / + (human colon cancer), or HCT116 p53-/- cells using FuGENE6 reagent. Cells were collected $48 \mathrm{~h}$ after transfection and subjected to SDS-PAGE and Western blotting. 


\section{Immunofluorescent staining}

A total of $1 \mu \mathrm{l}$ aliquots of pcDNA3.1 $(+) / \mathrm{N}-\mathrm{HA}-\mathrm{DUSP} 5$ were transfected into A549, HCT116 p53+/+, or HCT116 p53-/ - cells. At $48 \mathrm{~h}$ after transfection, cells were incubated in a medium containing MitoTracker for $30 \mathrm{~min}$, fixed in $4 \%$ paraformaldehyde for $1 \mathrm{~h}$, and treated with $0.1 \%$ Triton-X for $3 \mathrm{~min}$. After 1-h blocking in 3\% BSA, cells were incubated in $3 \%$ BSA containing anti-HAtag monoclonal antibody from rat and a secondary, fluorescein-labeled anti-rat antibody from rabbit for $1 \mathrm{~h}$. Nuclei were stained by DAPI, and cells were examined with a fluorescence microscope.

\section{Colony-formation assay}

Plasmids designed to express DUSP5 (pcDNA3.1(+ )/DUSP5) were constructed by cloning the entire coding region of DUSP5 cDNA into the pcDNA3.1(+) expression vector. The same sequence was inserted into the pcDNA3.1( - ) vector (pcDNA3.1(-)/DUSP5) to serve as a control. Nucleotide sequences of each construct were confirmed by sequencing. pcDNA3.1 $(+)$ vector was prepared as an additional control. H1299, HCT116 p53 + / +, and HCT116 p53-/- cells $\left(5 \times 10^{5}\right.$ each) were plated in $10-\mathrm{cm}$ culture dishes $24 \mathrm{~h}$ before transfection. $8 \mu \mathrm{g}$ of each expression vector (pcDNA3.1 $(+) /$

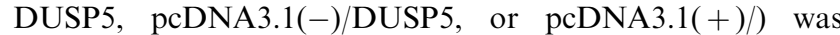
transfected using $48 \mu \mathrm{l}$ of FuGENE6 reagent; $48 \mathrm{~h}$ later, the harvested cells were diluted and replated in $10 \mathrm{~cm}$ culture

\section{References}

Brondello JM, Pouyssegur J and McKenzie FR. (1999). Science, 286, 2514-2517.

el-Deiry WS, Kern SE, Pietenpol JA, Kinzler KW and Vogelstein B. (1992). Nat. Genet., 1, 45-49.

Furuhata T, Tokino T, Urano T and Nakamura Y. (1996). Oncogene, 13, 1965-1970.

Guan KL and Butch E. (1995). J. Biol. Chem., 270, 7197-7203.

Hollstein M, Sidransky D, Vogelstein B and Harris CC. (1991). Science, 253, 49-53.

Iiizumi M, Arakawa H, Mori T, Ando A and Nakamura Y. (2002). Cancer Res., 62, 1246-1250.

Ishibashi T, Bottaro DP, Michieli P, Kelley CA and Aaronson SA. (1994). J. Biol. Chem., 269, 29897-29902.

Kim TI, Jin SH, Kang EH, Shin SK, Choi KY and Kim WH. (2002). Ann. N. Y. Acad. Sci., 973, 241-245.

Ko LJ and Prives C. (1996). Genes Dev., 10, 1054-1072.

Kovanen PE, Rosenwald A, Fu J, Hurt EM, Lam LT, Giltnane JM, Wright G, Staudt LM and Leonard WJ. (2002). J. Biol. Chem., 278, 5205-5213.

Levine AJ. (1997). Cell, 88, 323-331.

Martell KJ, Kwak S, Hakes DJ, Dixon JE and Trent JM. (1994). Genomics, 22, 462-464.

Marti F, Krause A, Post NH, Lyddane C, Dupont B, Sadelain $\mathrm{M}$ and King PD. (2001). J. Immunol., 166, 197-206.

McGrory WJ, Bautista DS and Graham FL. (1988). Virology, 163, 614-617.

Miyashita T and Reed JC. (1995). Cell, 80, 293-299.

Mori T, Anazawa Y, Matsui K, Fukuda S, Nakamura Y and Arakawa H. (2002a). Neoplasia, 4, 268-274.

Mori T, Anazawa Y, Iiizumi M, Fukuda S, Nakamura Y and Arakawa H. (2002b). Oncogene, 21, 2914-2918.

$\mathrm{Ng} \mathrm{CC}$, Koyama K, Okamura S, Kondoh H, Takei Y and Nakamura Y. (1999). Genes Chromosomes Cancer, 26, 329-335. dishes. Transfected cells were allowed to grow in the presence of $0.8 \mathrm{mg} / \mathrm{ml} \mathrm{G} 418$ (geneticin, Life Technologies) for 2 weeks. The colonies formed from each cell were fixed with $100 \%$ methanol, stained with crystal violet, and counted. To confirm the expression of DUSP5 in each dish, RT-PCR was constantly performed. The total RNA was extracted from cells with TRIzol reagent and reverse transcripted to cDNA. Then, PCR was performed to determine the quantity of DUSP5 transcript using primers; 5'-CTACCCACTCAACAGTCTCAGAGC- $3^{\prime}$ as a forward primer, and $5^{\prime}$ AAATCCCAGAAGTCCACAGCTT- $3^{\prime}$ as a reverse primer.

\section{Identification of phosphatase activity of DUSP5 toward Erk1/2}

pcDNA3.1(+)/DUSP5 expression vector was transfected into H1299 cells using Nucleofector (amaxa biosystems), cell line nucleofector kit V (amaxa biosystems), and nucleofector program T-20. pcDNA3.1(-)/DUSP5 vector was also transfected and used for control. In the same transfection protocol, we could transfect GFP expression vectors into more than $80 \%$ of H1299 cells (data not shown). Cells were collected 12, 24 , and $36 \mathrm{~h}$ after transfection, and their lysate was used for Western blotting. Western blotting was performed with $\mathrm{p} 44 / 42$ MAPK antibody or phospho-p44/42 MAPK (Thr202/ Tyr204) antibody (Cell Signaling) for primary antibody and goat antirabbit IgG-HRP sc-2004 (Santa Cruz Biotechnology) for secondary antibody.

Nishimori H, Shiratsuchi T, Urano T, Kimura Y, Kiyono K, Tatsumi K, Yoshida S, Ono M, Kuwano M, Nakamura Y and Tokino T. (1997). Oncogene, 15, 2145-2150.

Ochi K, Mori T, Toyama Y, Nakamura Y and Arakawa H. (2002). Neoplasia, 4, 82-87.

Oda K, Arakawa H, Tanaka T, Matsuda K, Tanikawa C, Mori T, Nishimori H, Tamai K, Tokino T, Nakamura Y and Taya Y. (2000). Cell, 102, 849-862.

Okamura S, Arakawa H, Tanaka T, Nakanishi H, Ng CC, Taya Y, Monden M and Nakamura Y. (2001). Mol. Cell, 8, 85-94.

Ono K, Tanaka T, Tsunoda T, Kitahara O, Kihara C, Okamoto A, Ochiai K, Takagi T and Nakamura Y. (2000). Cancer Res., 60, 5007-5011.

Oren M. (1994). Semin. Cancer Biol., 5, 221-227.

Shiraishi K, Fukuda S, Mori T, Matsuda K, Yamaguchi T, Tanikawa C, Ogawa M, Nakamura $\mathrm{Y}$ and Arakawa $\mathrm{H}$. (2000). Cancer Res., 60, 3722-3726.

Stefanovsky VY, Pelletier G, Hannan R, Gagnon-Kugler T, Rothblum LI and Moss T. (2001). Mol. Cell, 8, 1063-1073

Takei Y, Ishikawa S, Tokino T, Muto T and Nakamura Y. (1998). Genes Chromosomes Cancer, 23, 1-9.

Tanaka H, Arakawa H, Yamaguchi T, Shiraishi K, Fukuda S, Matsui K, Takei Y and Nakamura Y. (2000). Nature, 404, 42-49.

Tokino T, Thiagalingam S, el-Deiry WS, Waldman T, Kinzler K W and Vogelstein B. (1994). Hum. Mol. Genet., 3, 15371542 .

Urano T, Nishimori H, Han H, Furuhata T, Kimura Y, Nakamura Y and Tokino T. (1997). Cancer Res., 57, 3281-3287.

Yoo J, Park SY, Robinson RA, Kang SJ, Ahn WS and Kang CS. (2002). Arch. Pathol. Lab. Med., 126, 1096-1100. 